\title{
Reconciling probability theory and coherentism
}

\author{
Conal Duddy
}

Received: 4 March 2013 / Accepted: 20 June 2013 / Published online: 27 June 2013

(C) Springer Science+Business Media Dordrecht 2013

\begin{abstract}
Recent results in the literature appear to show that it is impossible for two independent testimonies to jointly raise the probability of a proposition if neither testimony individually has any impact on that probability. I show that these impossibility results do not apply when testimonies agree on incidental details.
\end{abstract}

Keywords Coherentism - Witness model · Probability theory - Coherence . Testimony $\cdot$ Bayesian

\section{Introduction}

According to the coherence theory of justification, a coherent set of multiple pieces of evidence may provide justification for a belief even though none of the pieces of evidence individually provides any justification for that belief. Among the most important contributions to this theory is Laurence BonJour's The structure of empirical knowledge 1985. ${ }^{1}$ Advocating the coherentist position, BonJour (pp. 147-148) appeals to the example given by Lewis (1946, p. 346) of 'relatively unreliable witnesses who independently tell the same circumstantial story'. Lewis uses this example to argue that coherence can amplify the credibility of the witnesses but, contrary to coherentist doctrine, he insists that each witness must have some initial degree of individual credibility that can be amplified. BonJour argues that coherence can generate credibility among testimonies where there was none.

Huemer (1997) provides a formal model in which BonJour's claims about the witness example collide with the rules of probability theory. Let $A$ denote a proposition,

${ }_{1}^{1}$ An excellent critique of coherentism is Erik J. Olsson's Against coherence 2005.

C. Duddy ( $\varangle)$

National University of Ireland Galway, Galway, Ireland

e-mail: conal.duddy@gmail.com 
and $\neg A$ its negation. There are two witnesses. Let $A_{1}$ be the proposition that the testimony of the first witness implies $A$ and let $A_{2}$ be the proposition that the testimony of the second witness implies $A$. Let us suppose that the witnesses do not influence one another and that neither witness alone has any impact upon our belief in $A$. Is it possible then that the conjunction of $A_{1}$ and $A_{2}$ would cause us to revise upwards the probability that $A$ is true? In other words, are conditions (1)-(3), as given below, mutually compatible?

(1) Witness independence: $P\left(A_{2} \mid A_{1}, A\right)=P\left(A_{2} \mid A\right)$

$P\left(A_{2} \mid A_{1}, \neg A\right)=P\left(A_{2} \mid \neg A\right)$

(2) Individual irrelevance: $P\left(A \mid A_{1}\right)=P(A)$

$P\left(A \mid A_{2}\right)=P(A)$

(3) Coherence justification: $P\left(A \mid A_{1}, A_{2}\right)>P(A)$

Huemer (1997) proves that these three conditions are not jointly compatible. Generalizations of this impossibility result are proved by Olsson (2005), Shogenji (2005) and Olsson and Shogenji (2004).

Olsson (2002) sets out a different witness model in his discussion of L. Jonathon Cohen's The probable and the provable $1977 .{ }^{2}$ Olsson and Shogenji (2004) and Olsson (2005) use that model to test Lewis's theses. Olsson's model features two propositions that are not in Huemer's model. One proposition is that both witnesses always tell the truth, and the other is that they always generate their reports at random. Prior probabilities are assigned to these propositions (summing to one). These probabilities can be updated when the witnesses give their testimonies.

An effect of these additional propositions is that the impact of corroboration becomes much more dramatic, particularly when witnesses agree on something previously thought improbable. Even if individual witnesses have just a slight degree of positive credibility, the probability of a proposition being true can rise rapidly as we hear cohering testimonies in support of it. However, without that slight degree of individual credibility this amplification cannot happen.

So both Huemer's and Olsson's models support what Olsson and Shogenji call Lewis's negative thesis that "if . . independent items [of evidence] are irrelevant to the hypothesis when taken singly, then they are also irrelevant when taken together however congruent they may be" (Olsson and Shogenji 2004). This presents a serious challenge to the theory of coherence justification.

\section{Reconciliation}

In two later contributions, Huemer $(2007,2011)$ argues that coherentists can escape from his previous impossibility result. Also, Shogenji (2005) demonstrates that recurrent coherence can generate justification from scratch. In this section I give a brief outline of some of the results contained in those papers.

$\overline{2}$ A variation of Olsson's model is proposed by Bovens et al. (2002). 


\subsection{Quasi independence}

Huemer (2007) proves that conditions (2) and (3) imply that $P\left(A_{2} \mid A_{1}, A\right)>P\left(A_{2} \mid\right.$ $\left.A_{1}, \neg A\right)$. Lets us denote this inequality by $\left(1^{*}\right)$. Huemer argues that the coherentist can replace condition (1) with $\left(1^{*}\right)$ and thereby circumvent the impossibility result. Huemer makes clear, however, that while this offers an escape from impossibility it does not vindicate the theory of coherence justification. To see why this is the case, consider the following scenario.

Suppose there are two witnesses to the landing of a fair coin. Let $A$ be the proposition that the coin landed heads up. The witnesses have agreed that if the coin lands heads up then they will either both lie (with a probability of $1 / 2$ ) or both tell the truth about which way the coin has landed. If the coin lands tails up then each witness will decide individually, at random and without bias, whether to lie or be truthful. Each testimony individually is worthless and yet if both witnesses testify that $A$ is true then the probability of $A$ rises from $1 / 2$ to $2 / 3$. Conditions $\left(1^{*}\right),(2)$ and (3) are satisfied, and even the second equation of condition (1) is satisfied. The problem is that this scenario is not at all like the example given by Lewis that BonJour appeals to, in which witnesses tell their stories independently. So BonJour's position is not vindicated by the compatibility of these conditions.

\subsection{Weak nonfoundationalism}

An interesting feature of the coin example given above is that if both witnesses report that the coin landed tails up then the probability that the coin landed tails up actually falls from $1 / 2$ to $1 / 3$. It turns out that this is not unique to the coin example. Huemer (2011) proves that conditions (2) and (3) imply that $P\left(A \mid \neg A_{1}, \neg A_{2}\right)>P(A)$. This inequality is called negative coherence justification and it is clearly not consistent with coherentism. However, Huemer argues that the coherentist can replace condition (2) with the requirement that $P\left(A \mid A_{1}, \neg A_{2}\right)=P(A)$. Let us call this condition $(2 *)$. The conjunction of $(2 *)$ together with $P\left(A \mid A_{1}, A_{2}\right)>P(A)$ does not imply $P\left(A \mid A_{1}\right)=P(A)$ or $P\left(A \mid A_{2}\right)=P(A)$ and so the coherentist is not committed to condition (2). Huemer is again clear that, while $\left(2^{*}\right)$ allows us to avoid $P\left(A \mid \neg A_{1}, \neg A_{2}\right)>P(A)$, this does not provide a vindication of coherence justification. This is true despite the fact that $(2 *)$ is not only compatible with (1*) and (3), but even with (1) and (3). Consider the following scenario.

Suppose again that there are two witnesses to the landing of a coin. As before, $A$ is the proposition that the coin landed heads up. In this scenario, each witness gives his or her report independently. Each witness tells the truth with a probability of $2 / 3$, lies with a probability of $1 / 3$ and is silent with a probability of zero. In this case, conditions (1), (2*) and (3) are all satisfied. It is clear, though, that each witness has some individual credibility since each one is more likely to tell the truth than to lie. So we cannot point to the compatibility of (1), $\left(2^{*}\right)$ and (3) as support for BonJour's position, although it does offer an escape from contradiction. 


\subsection{Recurring coherence}

Shogenji (2005) considers a case in which witnesses report on a sequence of events. Colored marbles are drawn randomly from an urn. Each witness always lies about the color of the marble drawn, always tells the truth or always randomizes. There are $n$ marbles and each witness is $n-1$ times more likely to be a liar than a truth teller. Suppose we hear two reports from each of two witnesses. Both witnesses assert that the first marble was blue and that the second was green. Shogenji shows that this raises the probability that the first marble was blue, even though neither report individually has any impact on that probability.

However, despite this result, Shogenji concludes, based on a result similar to Huemer's impossibility result, that "BonJour's intuitively plausible informal reasoning for [justification by coherence from scratch] is deficient". The case in which credibility is generated by recurrent coherence seems to differ from the witness scenario that BonJour refers to in that (i) the witnesses report on a sequence of separate events and (ii), as Shogenji demonstrates, the four reports that we considered above (two reports from each of two witnesses) are not conditionally independent relative to the first marble being blue.

My view is that Shogenji's finding, that recurrent coherence can generate justification from scratch, is very close to a vindication of BonJour's position on the witness scenario. I argue that via a combination of Huemer's and Shogenji's insights we can arrive at that vindication.

\section{Circumstantial stories}

Key to the intuitive plausibility of coherence justification in the witness scenario is that Lewis refers to witnesses telling the same circumstantial story. I take this to mean that the witnesses agree on details that are not essential but rather incidental to the proposition of primary significance. In order to take account of this feature of the witness scenario let us introduce three additional propositions to the formal model described in the introduction. Let $T$ be the circumstantial story. More formally, $T$ is a proposition that implies $A$, the proposition of primary significance, but is not implied by $A$. Let $T_{i}$ be the proposition that the testimony of witness $i$ implies $T$. So $P(A \mid T), P\left(A_{1} \mid T_{1}\right)$ and $P\left(A_{2} \mid T_{2}\right)$ are all equal to one.

Let us consider how the three conditions should be adapted to this model, beginning with condition (1).

\subsection{Witness independence}

As in the original condition (1), we will require that $A_{1}$ and $A_{2}$ are independent when the truth value of $A$ is fixed. They should also be independent when the truth value of $T$ is fixed. For brevity, let us denote this requirement by $A_{1} \perp A_{2} \mid A, \neg A, T, \neg T$. By this notation we mean that the two propositions on the left, separated by the symbol $\perp$, are conditionally independent relative to any one of the propositions that come after the vertical line. So, for example, condition (1) could be written as $A_{1} \perp A_{2} \mid A, \neg A$. 
We should similarly require that $A_{1}$ and $T_{2}$ are conditionally independent relative to each one of the four propositions $A, \neg A, T$ and $\neg T$. Of course, this goes for the pair $T_{1}$ and $A_{2}$ also.

However, I think that $T_{1}$ and $T_{2}$ should be required to be conditionally independent relative to each of the three propositions $T, \neg A$ and $A \wedge \neg T$ only. To see why, let us consider the following scenario.

Two individuals live in the same town but do not know each other. Each day they both decide whether to bring an umbrella to work. They are both influenced by whether it is raining and, if it is raining, whether that rain is light or heavy. There are not jointly influenced by any other factor. Let $R$ be the proposition that it is raining, and $H_{R}$ that there is heavy rain. Let $B_{1}$ be the event that Ann brings her umbrella, and $B_{2}$ that Bob brings his. The events $B_{1}$ and $B_{2}$ are not probabilistically independent since both individuals are influenced by the same weather. However, $B_{1}$ and $B_{2}$ are independent when it is given that $H_{R}$. They are also independent when it is given that $\neg R$. But we should not expect them to be independent when it is given that $R$. This is because both Ann and Bob are influenced by whether the rain is heavy or light and we are not controlling for this factor. Similarly, we should not expect $B_{1}$ and $B_{2}$ to be independent relative to $\neg H_{R}$ since both individuals are influenced by whether there is light rain or no rain at all. We can, however, expect $B_{1}$ and $B_{2}$ to be independent when it is given that $R \wedge \neg H_{R}$ (light rain).

Similarly, we should expect $T_{1}$ and $T_{2}$ to be conditionally independent relative to $T, \neg A$ and $A \wedge \neg T$, but not necessarily to $A$ or $\neg T$. If we require $T_{1}$ and $T_{2}$ to be conditionally independent relative to $A$ then we are in effect assuming that a witness's decision to assert $T$ is not affected by whether $A \wedge \neg T$ or $T$. This assumption does not seem reasonable. A requirement that $T_{1}$ and $T_{2}$ be conditionally independent relative to $\neg T$ implies that a witness's decision to assert $T$ is not affected by whether $A \wedge \neg T$ or $\neg A$. This seems unreasonable since, for example, a witness who is intent on lying about $A$ will consider telling story $T$ if $A$ is false, but will not report $T$ if $A$ is true. So condition (1) is adapted as follows.

(1**) Witness independence: $A_{1} \perp A_{2} \mid A, \neg A, T, \neg T$

$$
\begin{aligned}
& A_{1} \perp T_{2} \mid A, \neg A, T, \neg T \\
& T_{1} \perp A_{2} \mid A, \neg A, T, \neg T \\
& T_{1} \perp T_{2} \mid T, \neg A, A \wedge \neg T
\end{aligned}
$$

\subsection{Conditional credibility}

According to the coherence theory of justification, the addition of an item of evidence to a set of other items of evidence may raise the probability of a hypothesis supported by that set of evidence if the new item is coherent with the others. Suppose the set $\left\{A, T_{i}\right\}$ raises the probability of $T$ more than $A$ alone does. That is, $P\left(T \mid A, T_{i}\right)>$ $P(T \mid A)$. This would be consistent with coherence justification since $T_{i}$ is coherent with $A$. Credibility can be given to $T_{i}$ on the condition that it is accompanied by $A$ in the set of evidence. 
Let us suppose that the testimony of witness $i$ has no impact on the probability of $A$. That is, $P\left(A \mid T_{i}\right)=P(A)$. An implication of $P\left(A \mid T_{i}\right)=P(A)$ and $P(A \mid T)=1$ is that $P\left(T \mid A, T_{i}\right)>P(T \mid A)$ and $P\left(T \mid T_{i}\right)>P(T)$ are logically equivalent (see Claim 1 in the Appendix). Of course, $P\left(T \mid T_{i}\right)>P(T)$ is also consistent with the witness having some foundational, or intrinsic credibility. Crucially, however, if we attach some weight of credibility to the witness irrespective of coherence between the testimony of the witness and other evidence then we have not only $P\left(T \mid T_{i}\right)>P(T)$ but also $P\left(A \mid T_{i}\right)>P(A)$. Indeed, we may even expect the impact of $T_{i}$ on the probability of $A$ to be at least as great as its impact on the probability of $T$ since $A$ is the weaker proposition.

On the other hand, the conjunction of $P\left(T \mid T_{i}\right)>P(T)$ and $P\left(A \mid T_{i}\right)=P(A)$ is compatible with coherence justification. This would be the case if the degree of coherence between $T_{i}$ and any proposition weaker than $A$ were deemed to be insufficient for $T_{i}$ to derive any credibility, while the more impressive degree of coherence between $T_{i}$ and $A$ is considered sufficient.

Consider the following example. Let $\sigma$ denote an arbitrary sequence of six numbers. One morning I wake up and remember a vivid dream of a television lottery draw. So vivid that I can recall the sequence $\sigma$ of six numbers being drawn from the drum. Let $D_{6}$ be the proposition that I have this dream. Later in the day I watch the lottery draw on television. As each number is drawn I see that it matches with the sequence I dreamed of. It is only when the fifth number is drawn, matching the fifth number in my dream, that I am sufficiently impressed by the degree of congruence to raise the probability of the sixth number drawn being the sixth number in the sequence $\sigma$. Let $V_{5}$ and $V_{6}$ be the propositions that the first five and all six numbers, respectively, that I see being drawn match with the sequence $\sigma$. So we have $P\left(V_{6} \mid D_{6}, V_{5}\right)>P\left(V_{6} \mid V_{5}\right)$ and $P\left(V_{5} \mid D_{6}\right)=P\left(V_{5}\right)$ and $P\left(V_{5} \mid V_{6}\right)=1$. It follows that $P\left(V_{6} \mid D_{6}\right)>P\left(V_{6}\right)$. The inequality $P\left(V_{6} \mid D_{6}\right)>P\left(V_{6}\right)$ makes it appear as though I believe my dream to have some intrinsic degree of credibility. But this is not so, as is evidenced by $P\left(V_{5} \mid D_{6}\right)=P\left(V_{5}\right)$.

Requiring both $P\left(A \mid T_{i}\right)=P(A)$ and $P\left(T \mid T_{i}\right)=P(T)$ would mean that even if, hypothetically, we were to learn that much of what the witness reported is in fact true we would not give any credibility to the remainder of his or her testimony. I think that this goes beyond the case of a witness with "no antecedent degree of warrant or credibility" that BonJour (1985, p. 148) has in mind, and that we should require just $P\left(A \mid T_{i}\right)=P(A)$. The adaption of condition (2) then is as follows.

(2**) Individual irrelevance w.r.t. $A: P\left(A \mid T_{1}\right)=P(A)$

$$
P\left(A \mid T_{2}\right)=P(A)
$$

\subsection{Coherence justification}

I think that BonJour's claim about the witness scenario, when translated into the language of probability theory, is that conditions $\left(1^{* *}\right)$ and $\left(2^{* *}\right)$ are compatible with the following condition.

(3*) Coherence justification: $P\left(A \mid T_{1}, T_{2}\right)>P(A)$ 
The probability space illustrated in Fig. 1 shows that that $(1 * *),(2 * *)$ and $(3 *)$ are indeed mutually compatible. ${ }^{3}$ In that figure the large rectangle represents a sample space and each one of the 32 square cells represents an outcome. Each outcome is equally likely. The letter $A$ above a column indicates that the outcomes in that column are elements of $A$. So $A$ is the set of all 16 outcomes in the left half of the rectangle, and $T$ is the set of outcomes in the third and fourth columns. Similarly, $A_{1}$ is written next to a row to indicate that the outcomes in that row are elements of $A_{1}$, and so on. The elements of $T_{1}$ and $T_{2}$ are indicated by hatching and solid shading respectively.

Conditions (1**) and (2**) together with $P\left(T \mid A, T_{1}\right)>P(T \mid A)$ and $P(T \mid$ $\left.A, T_{2}\right)>P(T \mid A)$ jointly imply that condition $\left(3^{*}\right)$ is true (see Claim 2 in the Appendix).

Fig. 1 A probability distribution satisfying conditions $(1 * *),(2 * *)$ and $(3 *)$

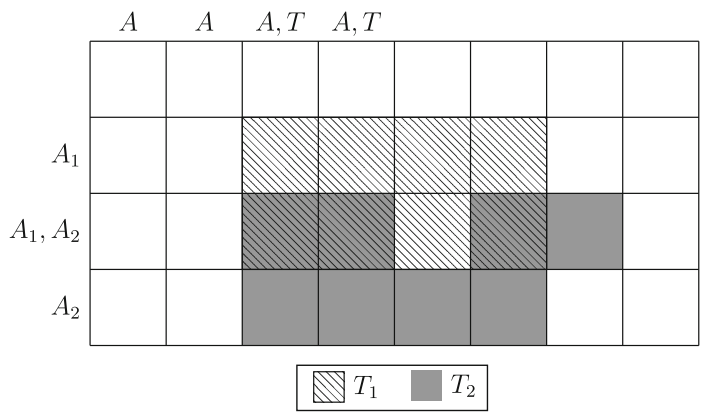

\subsection{Negative coherence justification}

As discussed above, Huemer (2011) proves that conditions (2) and (3) together imply $P\left(A \mid \neg A_{1}, \neg A_{2}\right)>P(A)$. This consequent is called negative coherence justification. By the same logical argument, conditions $\left(2^{* *}\right)$ and $\left(3^{*}\right)$ imply $P\left(A \mid \neg T_{1}, \neg T_{2}\right)>P(A)$. In Fig. 1 we have $P(A)=1 / 2$ and $P\left(A \mid \neg T_{1}, \neg T_{2}\right)=$ $10 / 19$. Note, however, that we do not have negative coherence justification since $P\left(A \mid \neg A_{1}, \neg A_{2}\right)=P(A)$.

While $P\left(A \mid \neg A_{1}, \neg A_{2}\right)>P(A)$ would be unacceptable, I think that $P(A \mid$ $\left.\neg T_{1}, \neg T_{2}\right)>P(A)$ is acceptable. In Fig. 1 we can see that the conjunction of $A_{1} \wedge A_{2}$ and $\neg T_{1} \wedge \neg T_{2}$ raises the probability of $A$ while $A_{1} \wedge A_{2}$ alone does not. This is consistent with coherence justification. Loosely speaking, adding $\neg T_{1} \wedge \neg T_{2}$ to $A_{1} \wedge A_{2}$ makes it more likely that both witnesses tell the same circumstantial story in support of $A$, though a story different from $T$. Suppose two witnesses agree that the defendant in a criminal trial was in a cafë on a particular afternoon. We ask each witness what

\footnotetext{
${ }^{3}$ Condition (1**) requires that $T_{1}$ and $T_{2}$ are conditionally independent relative to $A \wedge \neg T$, but, in Fig. 1, $P\left(T_{1} \wedge A \wedge \neg T\right)=0$ which means that $P\left(T_{2} \mid T_{1}, A, \neg T\right)$ is not defined and so we cannot claim that $P\left(T_{2} \mid T_{1}, A, \neg T\right)=P\left(T_{2} \mid A, \neg T\right)$. However, there is a more general, though less intuitive, definition of conditional independence. The more general definition requires $P\left(T_{1} \wedge T_{2} \mid A, \neg T\right)=$ $P\left(T_{1} \mid A, \neg T\right) P\left(T_{2} \mid A, \neg T\right)$. That equality does hold in Fig. 1.
} 
beverage the defendant ordered at the cafë, an incidental detail. If it is given that neither witness states that the defendant had tea then the probability that both witnesses state the same beverage is raised. It is because $\neg T_{1} \wedge \neg T_{2}$ raises the probability of the conjunction of $A_{1} \wedge A_{2}$ and $\neg T_{1} \wedge \neg T_{2}$ that we have $P\left(A \mid \neg T_{1}, \neg T_{2}\right)>P(A)$.

\section{Discussion}

We have seen that two independent items of evidence that are individually irrelevant to a given hypothesis can jointly raise the probability of that hypothesis if those items agree on incidental details. For example, suppose that I have a memory of a rock with a patch of moss being in a particular place. And I look at that place and see a rock with a patch of moss on it. I do not trust either this memory or current appearance. If I had knowledge of there being a rock in that location, though did not know whether it was mossy, then I would give some credibility to both the memory and current appearance of a rock with a patch of moss by virtue of their coherence with that knowledge. But I do not have knowledge of there being a rock and, moreover, neither my memory nor sense of sight provides me with any confirmation that there is a rock due to my mistrust in them. Yet, the combination of those two pieces of evidence, which I believe to be independent of one another, does provide me with some degree of confirmation that there is a rock.

Coherence among memories, perceptions and spontaneous beliefs can potentially confirm some of their contents to some degree without us having to bestow them with intrinsic credibility. We need only hold that they each would be deserving of credibility if we knew some substantial part of what they report to be accurate.

One weakness of the argument presented here is that I presuppose a method of assigning prior probabilities. How does one assign a prior probability to there being a rock in a particular place? This point is discussed by Huemer (2007). It may be that the kind of probabilistic analysis presented here is not appropriate for the question of epistemology being addressed. We must also ask how it is possible to establish independence among pieces of evidence. This question is addressed by Shogenji (2002).

Acknowledgments This work was supported by the Irish Research Council via a postdoctoral fellowship. I wrote this paper while visiting the Centre for Philosophy of Natural and Social Science at the London School of Economics and Political Science. I am grateful for their hospitality. I thank three anonymous reviewers for their insightful comments.

\section{Appendix}

Claim 1 Assume $P(A \mid T)=1$ and $P\left(A \mid T_{i}\right)=P(A)$. Then $P\left(T \mid A, T_{i}\right)>P(T \mid$ $A)$ if and only if $P\left(T \mid T_{i}\right)>P(T)$.

Proof We can write $P\left(T \mid A, T_{i}\right)>P(T \mid A)$ as

$$
\frac{P\left(T \wedge A \wedge T_{i}\right)}{P\left(A \wedge T_{i}\right)}>\frac{P(T \wedge A)}{P(A)}
$$


and, since $P(A \mid T)=1$, this can be rewritten as

$$
\frac{P\left(T \wedge T_{i}\right)}{P\left(A \wedge T_{i}\right)}>\frac{P(T)}{P(A)}
$$

which is equivalent to

$$
\frac{P\left(T_{i}\right) P\left(T \mid T_{i}\right)}{P\left(T_{i}\right) P\left(A \mid T_{i}\right)}>\frac{P(T)}{P(A)}
$$

and so we have

$$
\frac{P\left(T \mid T_{i}\right)}{P\left(A \mid T_{i}\right)}>\frac{P(T)}{P(A)} .
$$

Therefore, since $P\left(A \mid T_{i}\right)=P(A)$, we have

$$
P\left(T \mid T_{i}\right)>P(T) .
$$

So $P\left(T \mid A, T_{i}\right)>P(T \mid A)$ implies $P\left(T \mid T_{i}\right)>P(T)$, and by following the same steps in reverse order we can see that the implication goes in the other direction also.

Claim 2 Assume condition (2**) is true and $T_{1} \perp T_{2} \mid T, \neg A, A \wedge \neg T$ [the last line of condition $\left.\left(1^{* *}\right)\right]$ and $P(A \mid T)=1$. Then condition $\left(3^{*}\right)$ is true if and only if $P\left(T \mid T_{1}, A\right)$ and $P\left(T \mid T_{2}, A\right)$ are both strictly greater than, or both strictly less than, $P(T \mid A)$.

Proof The probability $P\left(T_{1} \wedge T_{2} \mid A\right)$ is equal to

$$
P\left(T_{1} \wedge T_{2} \mid A, \neg T\right) P(\neg T \mid A)+P\left(T_{1} \wedge T_{2} \mid T\right) P(T \mid A)
$$

and, given that $T_{1} \perp T_{2} \mid T, A \wedge \neg T$, we can rewrite this as

$$
P\left(T_{1} \mid A, \neg T\right) P\left(T_{2} \mid A, \neg T\right) P(\neg T \mid A)+P\left(T_{1} \mid T\right) P\left(T_{2} \mid T\right) P(T \mid A) .
$$

For brevity, let $x_{i}=P\left(T_{i} \mid A, \neg T\right), y_{i}=P\left(T_{i} \mid T\right)$ and $t=P(T \mid A)$. So we have

$$
P\left(T_{1} \wedge T_{2} \mid A\right)=(1-t) x_{1} x_{2}+t y_{1} y_{2}
$$

By definition, $T_{1} \perp T_{2} \mid \neg A$ implies that $P\left(T_{1} \wedge T_{2} \mid \neg A\right)$ equals $P\left(T_{1} \mid \neg A\right) P\left(T_{2} \mid\right.$ $\neg A)$. Condition (2**) implies that $P\left(T_{i} \mid \neg A\right)$ equals $P\left(T_{i} \mid A\right)$. So $P\left(T_{1} \wedge T_{2} \mid \neg A\right)$ is equal to $P\left(T_{1} \mid A\right) P\left(T_{2} \mid A\right)$. Note that $P\left(T_{i} \mid A\right)$ is equal to $\left((1-t) x_{i}+t y_{i}\right)$. So

$$
\begin{aligned}
P\left(T_{1} \wedge T_{2} \mid \neg A\right) & =\left((1-t) x_{1}+t y_{1}\right)\left((1-t) x_{2}+t y_{2}\right) \\
& =(1-t)^{2} x_{1} x_{2}+t^{2} y_{1} y_{2}+t(1-t)\left(x_{1} y_{2}+x_{2} y_{1}\right) .
\end{aligned}
$$


Hence, $P\left(T_{1} \wedge T_{2} \mid A\right)-P\left(T_{1} \wedge T_{2} \mid \neg A\right)$ is

$$
t(1-t)\left(x_{1} x_{2}+y_{1} y_{2}-x_{1} y_{2}-x_{2} y_{1}\right)
$$

or, equivalently,

$$
t(1-t)\left(y_{1}-x_{1}\right)\left(y_{2}-x_{2}\right) .
$$

We can see that $P\left(T_{1} \wedge T_{2} \mid A\right)-P\left(T_{1} \wedge T_{2} \mid \neg A\right)$ has the same sign as $\left(y_{1}-\right.$ $\left.x_{1}\right)\left(y_{2}-x_{2}\right)$. Therefore, $P\left(T_{1} \wedge T_{2} \mid A\right)>P\left(T_{1} \wedge T_{2} \mid \neg A\right)$ if and only if $\left(y_{1}>x_{1}\right.$ and $\left.y_{2}>x_{2}\right)$ or $\left(y_{1}<x_{1}\right.$ and $\left.y_{2}<x_{2}\right)$.

The inequality $P\left(T_{1} \wedge T_{2} \mid A\right)>P\left(T_{1} \wedge T_{2} \mid \neg A\right)$ is logically equivalent to $P\left(T_{1} \wedge\right.$ $\left.T_{2} \mid A\right)>P\left(T_{1} \wedge T_{2}\right)$, which is equivalent to $P\left(A \mid T_{1}, T_{1}\right)>P(A)$. And, since $T$ implies $A$, the inequality $y_{i}>x_{i}$ is equivalent to $P\left(T_{i} \mid T, A\right)>P\left(T_{i} \mid A, \neg T\right)$ and so also to $P\left(T_{i} \mid T, A\right)>P\left(T_{i} \mid A\right)$ and, therefore, to $P\left(T \mid T_{i}, A\right)>P(T \mid A)$. Similarly, $y_{i}<x_{i}$ is equivalent to $P\left(T \mid T_{i}, A\right)<P(T \mid A)$.

So we have $\left(A \mid T_{1}, T_{1}\right)>P(A)$ if and only if either $P\left(T \mid T_{i}, A\right)>P(T \mid A)$ for both $i \in\{1,2\}$ or $P\left(T \mid T_{i}, A\right)<P(T \mid A)$ for both $i \in\{1,2\}$.

\section{References}

BonJour, L. (1985). The structure of empirical knowledge. Cambridge, MA: Harvard University Press.

Bovens, L., Fitelson, B., Hartmann, S., \& Snyder, J. (2002). Too odd (not) to be true: A reply to Erik J. Olsson. British Journal for the Philosophy of Science, 53, 539-63.

Cohen, L. J. (1977). The probable and the provable. Oxford, MS: Clarendon Press.

Huemer, M. (1997). Probability and coherence justification. Southern Journal of Philosophy, 35, 463-72.

Huemer, M. (2007). Weak Bayesian coherentism. Synthese, 157, 337-346.

Huemer, M. (2011). Does probability theory refute coherentism? Journal of Philosophy, 108, 35-54.

Lewis, C. I. (1946). An analysis of knowledge and valuation. La Salle, IL: Open Court.

Olsson, E. J. (2002). Corroborating testimony, probability and surprise. British Journal for the Philosophy of Science, 53, 273-88.

Olsson, E. J. (2005). Against coherence: Truth, probability, and justification. Oxford, MS: Oxford University Press.

Olsson, E. J., \& Shogenji, T. (2004). Can we trust our memories? C.I. Lewis's coherence argument. Synthese, $142,21-41$.

Shogenji, T. (2002). The problem of independence in justification by coherence. In Y. Bouchard (Ed.), Perspectives on coherentism (pp. 129-137). Aylmer, QC: Éditions du Scribe.

Shogenji, T. (2005). Justification by coherence from scratch. Philosophical Studies, 125, 305-325. 